\title{
Estudo da Distribuição Espacial do Setor Agropecuário do Rio Grande do Sul
}

\section{Study of the Spatial Distribution of the Agricultural Sector in Rio Grande do Sul State}

Cláudia Bueno Rocha ${ }^{1}$

José Luiz Parré2

Resumo: Considerando a significativa importância da agropecuária à economia do Estado do Rio Grande do Sul, o presente trabalho objetivou analisar a distribuição espacial da mesma no ano de 2004, verificando a existência de agrupamentos (clusters), bem como observando o nível de correlação entre as variáveis analisadas na função de produção, cuja variável endógena é o valor bruto da produção agropecuária. Para tanto, utilizou-se como base metodológica a Análise Exploratória de Dados Espaciais (AEDE). Os resultados demonstraram a existência de autocorrelação espacial positiva entre os municípios e que os maiores valores da produção se encontram em municípios situados nas Regiões Norte e Nordeste. Através dos mapas de clusters verificou-se a presença de dois clusters do tipo baixo-baixo, situados ao Sul e Nordeste do Estado, e também três clusters do tipo alto-alto nas Regiões Norte e Nordeste, onde há o predomínio de pequenas propriedades, cultivo de uva e elevado valor bruto da produção.

Palavras-chave: Setor agropecuário. Rio Grande do Sul. Análise exploratória de dados espaciais.

Abstract: Considering the significant importance of agriculture for the State of Rio Grande do Sul economy, the present work aimed to examine the spatial distribution of agriculture in the State of Rio Grande do Sul in 2004, verifying the existence of clusters, as well as to analyze the level of correlation among the variables analyzed in the production function, using as endogenous variable the gross value of agricultural production. Thus, the Exploratory Analysis of Spatial Data was used as methodological basis. The results demonstrated the existence of positive spatial autocorrelation between towns and that the greatest values of production are found in towns situated in the North and Southeast regions. The maps of clusters allowed to verify the existence of two low-low clusters,

1 Graduada em Ciências Econômicas pela Universidade Estadual de Maringá (UEM) e mestranda em Economia Aplicada pela Escola Superior de Agricultura "Luiz de Queiroz" (ESALQ/USP). E-mail: clabu_rocha@yahoo.com.br

2 Professor Associado do Departamento de Economia da Universidade Estadual de Maringá (UEM). E-mail: jlparre@uem.br 
situated in the South and Northeast of the state, and three high-high clusters in the North and Northeast region, where there is predominance of small properties, grape cultivation and high gross value of production.

Keywords: Agricultural sector. Rio Grande do Sul. Exploratory analysis of spatial data.

JEL Classification: R12; Q19.

\section{Introdução}

Inserida no processo de abertura econômica, intensificado na década de 1990, a estrutura agropecuária do Estado do Rio Grande do Sul vem sofrendo consideráveis transformações. Com o propósito de aumento da competitividade interna e externa, a diversificação na pauta de produção e incorporação de novas tecnologias se tornaram mecanismos essenciais, uma vez que o desenvolvimento das atividades econômicas dependem da adequação às constantes mudanças no ambiente comercial.

O setor agropecuário pode ser considerado elemento estratégico para a consolidação do programa de estabilização da economia brasileira, devido ao grande efeito multiplicador do complexo agroindustrial no Produto Interno Bruto (PIB), assim como devido ao peso dos produtos agrícolas na pauta de exportações e a contribuição desse setor para o controle da inflação (SOUZA e SILVA, 2004).

Considerando a importância da agropecuária para o Estado, o estudo da função de produção agropecuária faz-se necessário pois, segundo Gomes e Baptista (2004), esse estudo é um importante instrumental para análise desse setor. A estimação e interpretação da função auxiliam a tomada de decisão e a alocação dos recursos escassos, melhorando assim a produção agropecuária da região.

A função agropecuária, de acordo com Simm e Alves (2005), por sua própria característica encontra-se distribuída ao longo de todo território, sendo associada a fatores edafoclimáticos, culturais e ambientais. Além disso, a produção de insumos e o processamento dos produtos de origem agropecuária podem apresentar características bem definidas em termos de localização e, em muitos casos, ser influenciados fortemente pela localização da fonte de matéria prima.

O fenômeno espacial é muito presente na agropecuária pois, segundo Almeida (2005), a dependência espacial é facilmente verificável nesse setor, uma vez que os produtores rurais têm facilidade em observar o que os vizinhos estão produzindo. Ademais, essas culturas dependem de recursos naturais concentrados no espaço geográfico, reforçando o efeito de que a produção ocorre na forma de agrupamentos (clusters), espalhados pelo espaço econômico. 
Deve-se destacar ainda que estudos relacionados ao setor primário apresentam alta relevância para a sociedade, visto que esse desempenha um importante papel no desenvolvimento econômico, exercendo fortes efeitos de encadeamento no restante da economia.

Diante de tais considerações, o presente estudo objetiva analisar a distribuição espacial da agropecuária no Estado do Rio Grande do Sul no ano de 2004, verificando a existência de clusters, bem como examinar o nível de correlação entre as variáveis contidas na função de produção.

\section{Estado do Rio Grande do Sul}

Considerada a quarta economia do Brasil em termos de PIB no ano de 2007, de acordo com a Fundação de Economia e Estatística (FEE), o Estado do Rio Grande do Sul apresenta-se como fundamental ao desenvolvimento econômico do país (FEE, 2008). O Estado é composto por 496 municípios e possui população de 10.845.087 habitantes distribuídos numa área de $281.749 \mathrm{Km}^{2}$ (IBGE, 2008).

Representando aproximadamente $11 \%$ do Valor Adicionado Bruto (VAB) da economia gaúcha, segundo a FEE, a agropecuária foi a grande responsável pelo bom desempenho econômico do Estado no ano de 2007 e, apesar de sua baixa participação, comparada aos demais segmentos da economia, o setor apresentou a maior taxa de crescimento no ano de 2007, bastante superior à taxa observada no Brasil (Tabela 1).

Tabela 1 - Taxas de Crescimento do VAB por setores de atividade e do PIB do Rio Grande do Sul e do Brasil - 2007 (\%)

\begin{tabular}{c|c|c}
\hline Discriminação & Rio Grande do Sul & Brasil $^{1}$ \\
\hline Agropecuária & 19,2 & 4,3 \\
\hline Indústria & 7,2 & 6,0 \\
\hline Serviços & 5,2 & 4,7 \\
\hline PIB & 7,0 & 5,2 \\
\hline
\end{tabular}

${ }^{1}$ Estimativa para o ano.

Fonte: FEE/Centro de Informações Estatísticas/Núcleo de Contabilidade Social (2008). IPEA - Boletim de Conjuntura - Dez. 2007.

O agronegócio é tido como o principal motor de crescimento gaúcho, uma vez que possibilita a dinâmica das exportações, principalmente de produtos derivados de soja, como óleo de soja, fumo, tratores e carnes de frango (FEE, 2008). 


\subsection{O Setor Agropecuário Gaúcho}

O setor agropecuário gaúcho apresenta características bastante diversificadas quando observadas as diferentes localidades do Estado. De acordo com Alonso et al. (1994), o Estado pode ser dividido em três Regiões: a Sul, onde predominam a grande propriedade, a pecuária e a lavoura de arroz; a Norte, predominantemente agrária, caracterizada pelas pequenas e médias propriedades, e onde a produção inicialmente diversificada e com utilização de mão-de-obra familiar tem cedido espaço para as lavouras mecanizadas de trigo e soja; e a última região, a Nordeste, que se caracteriza pela presença de vários setores industriais, além de grandes concentrações urbanas.

Destaca-se, segundo Batista et al. (2006), que as pequenas propriedades, localizadas na Região Norte e que possuem condições de solo e relevo menos favoráveis, vêm apresentando restrições a práticas agrícolas intensivas como o uso de tecnologias motomecânicas. Dessa forma, a Região Norte tem convivido com o aumento do tamanho da propriedade e a concentração de terra em detrimento de pequenos produtores.

Na Região Sul, por sua vez, o que se observa é o desenvolvimento predominante da pecuária extensiva e da agricultura. É importante ressaltar que em várias localidades da Região Sul, as condições de solo não permitem uma maior diversificação das atividades, devido à suscetibilidade à erosão que algumas áreas apresentam, enquanto outras não são ideais para uso de maquinários e implantação de lavouras temporárias.

Valendo-se da vantagem de se localizar próxima a grandes concentrações urbanas, a Região Nordeste tem no comércio e na indústria a base para seu desenvolvimento econômico.

De acordo com a Secretaria do Planejamento e Gestão do Rio Grande do Sul (SEPLAG), na estrutura do valor bruto da produção agropecuária destaca-se a lavoura, a qual representa $61,3 \%$ da produção, seguida pela produção animal com 34,0\%. Dentre os diferentes tipos de lavoura, a produção de grãos é a mais significativa (36,4\% em 2004). Ressalta-se também que, do total de estabelecimentos do Estado, 85,7\% possuem menos de 50 hectares, os quais ocupam apenas $24,4 \%$ da área total utilizada pela agropecuária; e as propriedades com mais de 500 hectares representam $1,83 \%$ dos estabelecimentos, ocupando $41,66 \%$ da área rural, o que demonstra a concentrada estrutura fundiária do Estado (SEPLAG, 2008).

\section{Fundamentos Metodológicos}

O presente trabalho utiliza como base metodológica técnicas de distribuição espacial, tendo início com a Análise Exploratória de Dados Espaciais (AEDE). 


\subsection{Análise Exploratória dos Dados Espaciais}

A análise exploratória de dados espaciais (AEDE), segundo Perobelli et al. (2005), está baseada nos aspectos espaciais contidos na base dos dados. Assim, considera tanto a dependência espacial quanto a heterogeneidade espacial. O objetivo desse método é descrever a distribuição espacial, os padrões de associação espacial (clusters espaciais), verificar a presença de diferentes regimes espaciais ou outras formas de instabilidade espacial (não estacionariedade) e identificar outiliers.

É importante ressaltar, segundo Almeida (2004), que essa análise é mais apropriada na investigação de variáveis espacialmente densas ou intensivas, isso é, variáveis que são divididas por algum indicador de intensidade (variáveis per capita ou por área, ou ainda as divididas pela quantidade de capital ou trabalho). A relevância é que essas variáveis estariam considerando as externalidades relevantes na consideração do fenômeno estudado (efeitos de aglomeração, de vizinhança e/ou congestão).

A partir da AEDE é possível extrair medidas de autocorrelação espacial global elocal, investigando a influência dos efeitos espaciais por intermédio de métodos quantitativos.

De acordo com Almeida (2004), a AEDE é tida como pré-requisito ao estudo da econometria espacial, subcampo da econometria que lida com as complicações causadas pela interação espacial (autocorrelação espacial) e pela estrutura espacial (heterogeneidade espacial) em modelos de regressão para dados na forma de cross-section e painel de dados. Sendo assim, mostra-se importante na identificação de observações discrepantes no espaço e possíveis padrões de associação espacial.

\subsubsection{Autocorrelação Espacial Global}

O estudo da AEDE inicia-se verificando a aleatoriedade dos dados espaciais, significando que os valores do atributo numa região não dependem dos valores desse atributo nas regiões vizinhas.

Segundo Perobelli et al. (2005), a autocorrelação espacial pode ser calculada pela estatística I de Moran. Através dela obtém-se a indicação formal do grau de associação linear entre os vetores de valores observados no tempo $t\left(z_{t}\right)$ e a média ponderada dos valores da vizinhança, ou os lags espaciais $\left(W z_{t}\right)$. Os valores de I maiores (ou menores) do que o valor esperado $\mathrm{E}(\mathrm{I})=-1 /(\mathrm{n}-1)$ demonstram a existência de autocorrelação positiva (ou negativa).

A autocorrelação espacial positiva, de acordo com Almeida (2004), revela a existência de uma similaridade entre os valores do atributo estudado e da localização espacial desse. A autocorrelação espacial negativa revela, por sua vez, que existe uma dissimilaridade entre os valores do atributo considerado e sua localização espacial. 
A estatística I de Moran pode ser expressa como:

$$
I_{t}=\left(n / S_{0}\right)\left(z_{t}^{\prime} W z_{t} / z_{t}^{\prime} z_{t}\right) \mathrm{t}=1, \ldots, \mathrm{n}
$$

A normalização das linhas da matriz de pesos espaciais, ou seja, quando os elementos de cada linha somam 1, altera a expressão (1) para a seguinte forma:

$$
I_{t}=\left(z_{t}^{\prime} W z_{t} / z_{t} z_{t}\right) \mathrm{t}=1, \ldots, \mathrm{n}
$$

É de grande relevância destacar que a estatística I de Moran é uma medida global, portanto, não é possível observar a estrutura de correlação espacial regional.

\subsubsection{Autocorrelação Espacial Local}

A estatística global do I de Moran, segundo Perobelli et al. (2005), pode esconder padrões locais de autocorrelação espacial, sendo possível ocorrer três situações distintas. A primeira envolve o indício de um I de Moran global insignificante, podendo ocorrer indicações de autocorrelação espacial com a mesma característica. A segunda incorre em um I de Moran global que oculta autocorrelação espacial local negativa e insignificante; e a terceira indica que a evidência de uma autocorrelação espacial global negativa pode acomodar indícios de autocorrelação espacial local positiva para certos grupos de dados. Por isso é importante avaliar o padrão local de autocorrelação espacial a fim de se obter um maior detalhamento dos resultados.

Em 1995 foi sugerido por Anselin um novo indicador com a capacidade de observar os padrões locais de associação linear estatisticamente significantes. De acordo com Almeida (2005), o indicador de I de Moran local faz a decomposição do indicador global de autocorrelação na contribuição local de cada observação em quatro categorias, cada uma individualmente correspondendo a um quadrante no diagrama de dispersão de Moran.

A estatística I de Moran local pode ser obtida pela seguinte fórmula:

$$
I_{i}=\frac{\left(y_{i}-\bar{y}\right) \sum_{j} w_{i j}\left(y_{j}-\bar{y}\right)}{\sum_{i}\left(y_{i}-\bar{y}\right)^{2} / n}
$$


Sob o pressuposto da aleatorização, o valor esperado da estatística I de Moran local é dado por: $\mathrm{E}$ (Ii) = -wi/(n-1), em que wi é o somatório dos elementos da linha.

\subsubsection{Digramas de Dispersão de Moran}

O diagrama de dispersão de Moran, de acordo com Perobelli et al. (2005), é uma das formas de interpretar a estatística I de Moran. Através da representação do coeficiente de regressão, é possível visualizar a correlação linear entre $z$ e Wz no gráfico que considera duas variáveis. No caso específico da estatística I de Moran tem-se o gráfico de Wz e z. Portanto, o coeficiente I de Moran é dado pela inclinação da curva de regressão de Wz contra $z$ e essa apresentará o grau de ajustamento.

O digrama de dispersão de Moran, de acordo com Almeida (2004), é divido em quatro quadrantes, os quais correspondem a quatro padrões de associação local espacial entre as regiões e seus vizinhos. $\mathrm{O}$ primeiro quadrante (localizado na parte superior direita) mostra as regiões que apresentam altos valores para a variável em análise cercadas por regiões que também apresentam valores acima da média para a mesma variável. Esse quadrante é classificado como alto-alto (AA). O segundo quadrante (localizado na parte superior esquerda) mostra as regiões com baixos valores para a variável em análise cercadas por vizinhos que apresentam valores altos. Tal quadrante é classificado como baixo-alto (BA). $\mathrm{O}$ terceiro quadrante (localizado no canto inferior esquerdo) é constituído pelas regiões cujos valores para a variável em análise são baixos e são circundadas por regiões que também possuem baixos valores, e é classificado como baixo-baixo (BB). O último e quarto quadrante (localizado no canto inferior direito) é formado pelas regiões com altos valores para as variáveis em analise e que são circundadas por regiões de baixos valores. Esse quadrante é classificado como alto-baixo (AB).

As regiões que estão localizadas nos quadrantes $\mathrm{AA}$ e $\mathrm{BB}$ apresentam autocorrelação espacial positiva, ou seja, formam clusters de valores similares. Por outro lado, os quadrantes $\mathrm{BA}$ e $\mathrm{AB}$ apresentam autocorrelação espacial negativa, formando clusters com valores diferentes.

\subsection{Descrição das Variáveis e Fontes de Dados}

Neste estudo, procurou-se considerar as variáveis que permitissem captar com maior precisão a composição da função de produção agropecuária do Rio Grande do Sul. Além das variáveis tradicionais - capital, trabalho e área - foi inserida a variável eletricidade como proxy para captar o grau de modernização da agropecuária do Estado. Levou-se também em consideração as diferenças edafoclimáticas dos municípios, inserindose, assim, a densidade pluviométrica (chuva) e a temperatura municipal. 
A variável dependente ( $v b p$ ) é o valor bruto da produção agropecuária dos municípios, medido em reais, para o ano 2004, e foi obtido junto à Fundação de Economia e Estatística do Rio Grande do Sul (FEE). A variável trabalho (trab), representada pelo número de pessoas ocupadas nos estabelecimentos agropecuários, foi fornecida pela Relação Anual de Informações Sociais (RAIS). A variável capital (cap) é representada pelo valor total do estoque de crédito rural e foi obtida junto ao Instituto de Pesquisa Econômica Aplicada (IPEA). A variável área (area), representada pela área colhida de lavoura tanto permanente quanto temporária, foi fornecida pela Pesquisa Agrícola Municipal (PAM), realizada pelo IBGE. $\mathrm{O}$ insumo energia elétrica (ene), que corresponde à média do consumo de energia elétrica rural em megawatt por município, foi obtido junto à FEE. A variável climática precipitação total anual (chu), em mm, foi fornecida pela Secretaria de Infra-Estrutura e Logística do Rio Grande do Sul (SEMC). A variável categórica temperatura média anual (temp), em graus centígrados, assume nove valores, conforme faixa de temperatura, sendo o valor 1 para a faixa de temperatura entre 8 e 10 graus centígrados; o valor 2 para 10 a 12 graus centígrados; o valor 3 para 12 a 14 graus centígrados; seguindo a classificação até o valor 9, que corresponde ao intervalo entre 24 e 26 graus centígrados. Essa variável também foi obtida junto à SEMC.

Os conjuntos dos dados são do tipo seção cruzada (cross-section) para os municípios do Estado do Rio Grande do Sul; sendo assim, a amostra é composta por 496 observações. As variáveis vbp, trab, area, cap e ene são intensificadas, ou seja, são divididas pela área territorial medida em $\mathrm{Km} 2$, uma vez que variáveis absolutas ou extensivas poderiam levar ao engano na interpretação dos resultados. Já as variáveis chu e temp são categóricas.

\subsection{Função de Produção}

A função de produção utilizada neste trabalho é do tipo CobbDouglas pois, segundo Castro (2002), é a mais utilizada, uma vez que sua interpretação é facilmente realizada e apresenta boa qualidade estatística.

Dessa forma, este modelo geral apresenta-se da seguinte forma:

$$
v b p_{t}=f\left(\text { trab }_{t}, \text { cap }_{t}, \text { area }_{t}, \text { ene }_{t}, \text { chu }_{t}, \text { temp }_{t}\right)
$$

em que,

$\mathrm{t}=1,2,3, \ldots ., 496$ são os municípios. 


\section{Resultados e Discussões}

\subsection{Análise Exploratória dos Dados Espaciais (AEDE)}

O presente trabalho teve início com a análise das variáveis utilizadas no estudo da função de produção da agropecuária do Estado do Rio Grande do Sul, juntamente com a verificação da distribuição espacial do setor. Para tanto, utilizou-se a AEDE, visto que essa busca analisar estatisticamente informações geográficas como forma de descobrir padrões de associação espacial. As variáveis foram intensificadas pela área territorial dos municípios como forma de extinguir externalidades que poderiam levar a resultados falazes.

A distribuição do valor bruto da produção, como pode ser observada através do Mapa 1, não é homogênea entre os municípios. As regiões que apresentam a maior concentração de municípios com elevado valor da produção são a Norte e a e a Nordeste. ${ }^{3}$ Situados na região Nordeste, região serrana do Estado, os municípios de Venâncio Aires, Vera Cruz, Santa Cruz do Sul, Boqueirão do Leão, Flores da Cunha, São Valentim do Sul, Monte Belo do Sul, Teutônia, Bento Gonçalves, Garibaldi, Farroupilha, dentre outros, são caracterizados como municípios pequenos, desenvolvidos e relativamente rurais. Têm como principais atividades agropecuárias o cultivo de uva, a criação de aves e animais de pequeno porte, assim como o cultivo de milho, porém em menor proporção (JANSEN; WAQUIL, 2002).

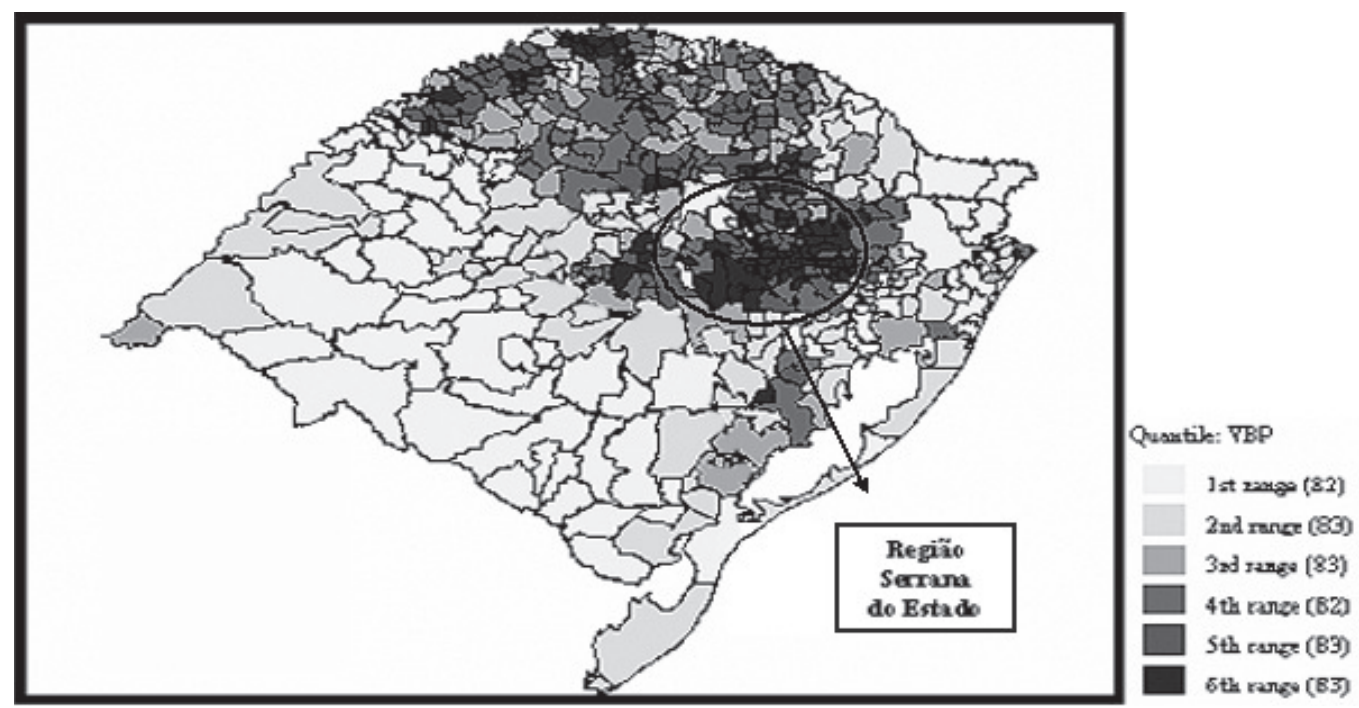

Mapa 1 - Distribuição do valor bruto da produção agropecuária do Rio Grande do Sul no ano de 2004 (Mil reais/Km2)

Fonte: Elaboração dos autores.

3 Regiões do Rio Grande do Sul baseadas na divisão elaborada por Alonso et al. (1994), a qual considera o estado dividido em apenas três regiões: Norte, Nordeste e Sul. 
Deve-se destacar ainda a presença de municípios com elevado VBP no Norte do Estado, os quais se caracterizam pela diversidade na produção agropecuária. Dentre os produtos de maior relevância estão o fumo, a mandioca, a batata-inglesa, a laranja, a banana, a erva-mate, assim como produtos provenientes da horticultura e floricultura.

Embora estejam presentes no Norte do Estado municípios com alto valor da produção, cabe salientar que, segundo Jansen e Waquil (2002), essa região se caracteriza pela presença de pequenos municípios, predominantemente rurais e relativamente pobres.

Ao analisar o valor bruto da produção, levando-se em consideração a presença de municípios tidos como outliers, ou seja, municípios com valores discrepantes do restante do Estado, verifica-se a presença de 11 municípios que não seguem o mesmo processo de dependência espacial dos demais: 5 considerados outliers altos e 6 outliers baixos. Dessa forma, exercem uma influência espúria sobre a medida global de autocorrelação (Mapa 2). De acordo com Almeida (2004), uma observação é considerada outlier global superior (inferior) quando se situa acima (abaixo) da fronteira superior (inferior) do intervalo interquartílico em uma quantidade no mínimo superior 1,5 vezes ao valor do intervalo.

Os municípios considerados outliers altos, destacados no Mapa 2, situam-se nas regiões Norte e Nordeste do Estado e são caracterizados pela pequena dimensão territorial e pelo elevado valor da produção agropecuária.

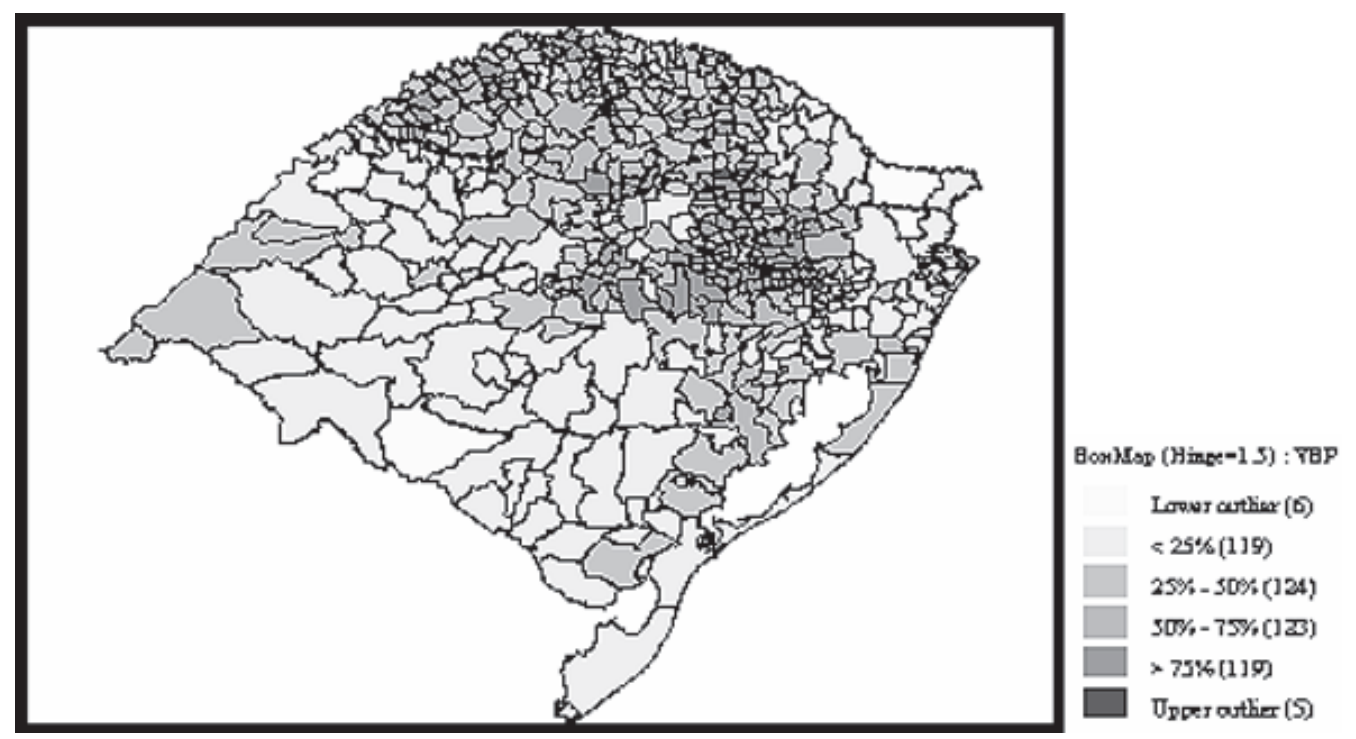

Mapa 2 - Valor bruto da produção agropecuária do Rio Grande do Sul (Mil reais/Km2) considerando a presença de municípios outlier

Fonte: Elaboração dos autores.

Uma outra ferramenta que pode ser utilizada na detecção de outliers de nível superior e inferior é o cartograma que, segundo Almeida (2004), 
mostra-se como um mapa em que os polígonos irregulares que representam os municípios são substituídos por círculos de tamanho proporcional ao valor da variável considerada. Os círculos podem ser de cor cinza claro, cinza escuro ou preta, de tal forma que os círculos de cor cinza claro seguem o mesmo padrão quanto ao valor da produção, os círculos cinza escuro são valores elevados, sendo muito discrepantes segundo o critério 1,5 hidge, ${ }^{4}$ e os círculos pretos são valores baixos muito discrepantes.

Confirmando a presença de municípios com elevados valores da produção agropecuária, o cartograma (Figura 1) evidencia a existência de quatro municípios considerados outliers altos: Vale Real, Lindolfo Collor, Ivoti e Dom Pedro de Alcântara, os quais, apesar da pequena extensão territorial, possuem elevado valor de produção.

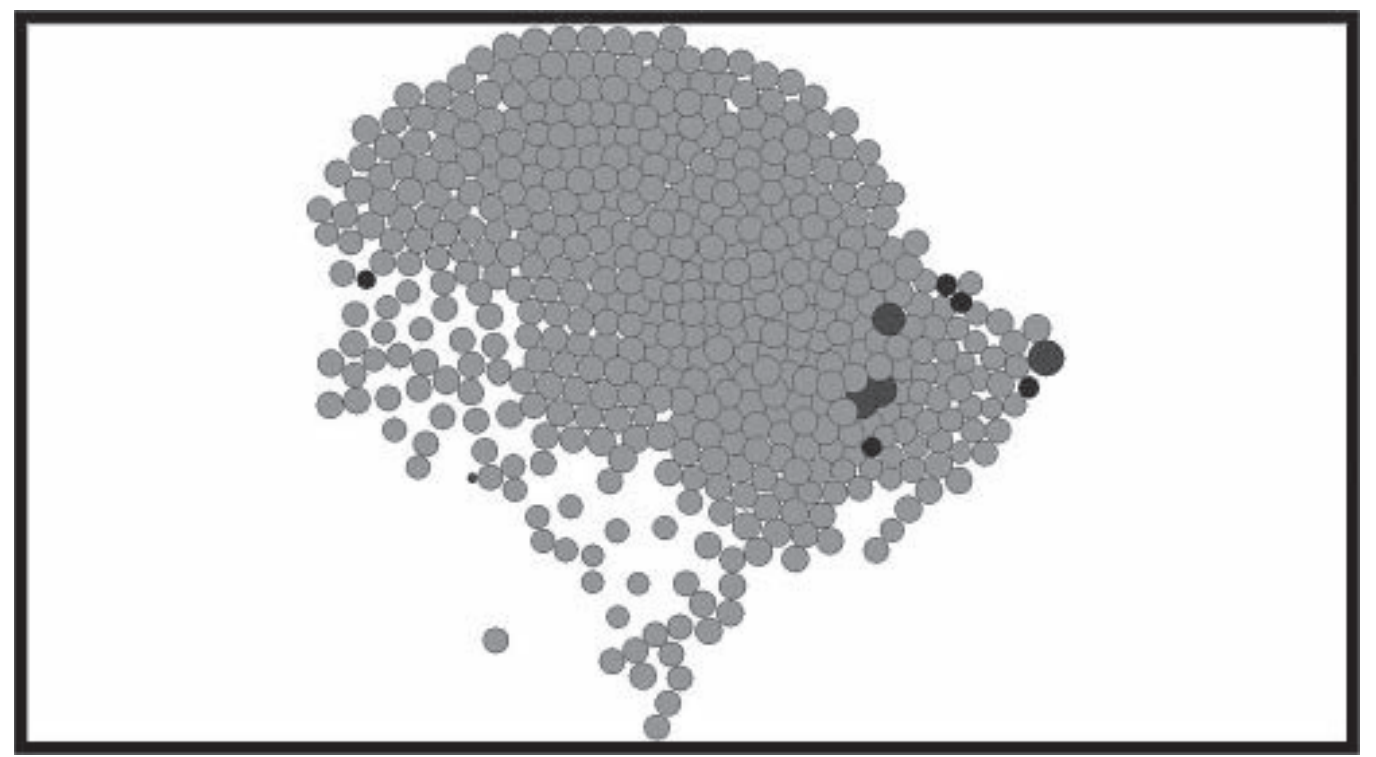

Figura 1 - Cartograma para o valor bruto da produção agropecuária do Rio Grande do Sul

Fonte: Elaboração dos autores.

A visualização de mapas mostra-se importante na verificação do comportamento da variável estudada em determinada região, todavia, apenas a conferência visual das figuras pode induzir ao erro. Por conseguinte, para a confirmação dos resultados obtidos através dos mapas, é necessária a realização de testes de aleatoriedade, ou seja, verificar a tendência geral dos agrupamentos dos dados.

\subsubsection{I de Moran Global}

Uma forma alternativa de visualizar a associação espacial de um determinado fenômeno é através do diagrama de dispersão de Moran,

4 De acordo com Almeida (2004), 1,5 hidge significa que a observação aparece fora da fronteira do intervalo interquartílico em um montante que é, no mínimo, 1,5 vezes o valor do intervalo interquartílico. 
o qual fornece relevantes informações sobre o grau de dependência espacial. De acordo com Pimentel, Almeida e Sabbadini (2005), os valores que excedem o I de Moran calculado indicam que há autocorrelação espacial positiva, enquanto que valores abaixo do esperado indicam a presença de autocorrelação negativa. A autocorrelação positiva evidencia uma similaridade entre os valores da variável estudada e a localização espacial da variável. Portanto, a autocorrelação negativa indica a existência de uma dissimilaridade entre os valores da variável estudada e a localização dessa.

Considerando a variável endógena ao modelo, o valor bruto da produção agropecuária, na ocorrência de autocorrelação positiva, significa que os municípios que possuem alto (baixo) valor da produção são circundados por municípios que também possuem valores altos (baixos) para essa variável. Logo, quando o I de Moran detecta autocorrelação negativa, denota que os municípios com alto (baixo) valor bruto da produção são circundados por municípios com baixo (alto) valor para essa variável.

O I de Moran esperado, $\mathrm{E}(\mathrm{I})=-1 /(\mathrm{n}-1)$, isso é, o valor que seria obtido caso não houvesse padrão espacial nos dados, é -0,002. Dessa forma, os valores de I acima do I de Moran esperado indicam autocorrelação espacial positiva e os dados abaixo indicam autocorrelação negativa.

Analisando quatro diferentes tipos de matrizes de pesos espaciais - 5 vizinhos, 6 vizinhos, torre e rainha - são apresentados os valores de I de Moran para a variável valor bruto da produção agropecuária (Tabela 2). Como se verifica, há indício de autocorrelação positiva entre os municípios ao nível de significância estatística de 1\% pois, para todas as convenções, o valor de I está acima do valor esperado da estatística.

Tabela 2 - Coeficiente de I de Moran para o valor bruto da produção agropecuária do Rio Grande do Sul

\begin{tabular}{c|c|c}
\hline Convenção & I & Probabilidade \\
\hline 5 vizinhos & 0,5859 & 0,01 \\
\hline 6 vizinhos & 0,5795 & 0,01 \\
\hline Torre & 0,6050 & 0,01 \\
\hline Rainha & 0,5889 & 0,01 \\
\hline
\end{tabular}

* a pseudossignificância empírica é baseada em 999 permutações aleatórias. Fonte: Elaboração dos autores.

A Figura 2 apresenta os diagramas de dispersão de Moran para os quatro tipos de matrizes de pesos consideradas. Os valores apresentados na Tabela 2, juntamente à dispersão dos municípios nas figuras, enfatizam a existência de autocorrelação espacial positiva para o valor bruto da produção agropecuária do Rio Grande do Sul. Os pontos que 
representam os municípios, em todas as figuras, se concentram no primeiro e no terceiro quadrante.

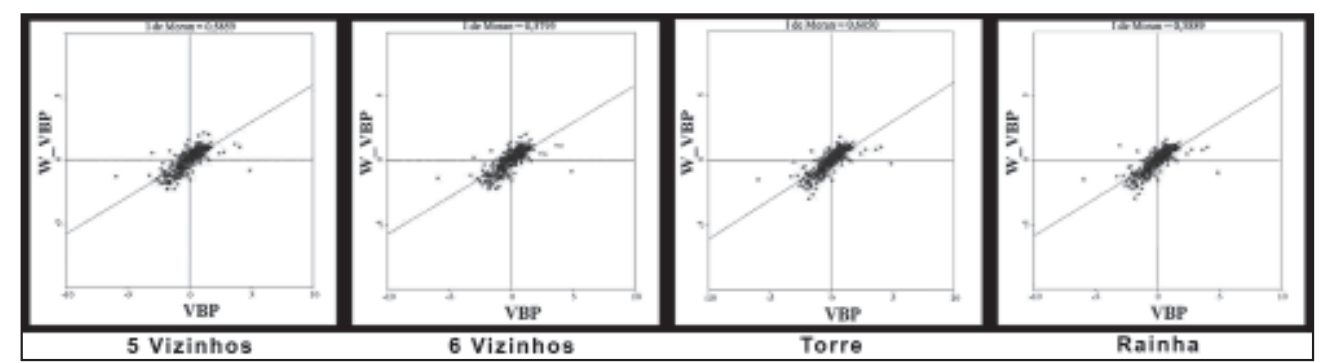

Figura 2 - Diagramas de dispersão de Moran para o valor bruto da produção agropecuária (Mil reais/Km2) do Rio Grande do Sul

Fonte: Elaboração dos autores.

É relevante destacar, segundo Perobelli et al. (2005), que para haver a confirmação da autocorrelação espacial é necessário que a inclinação da curva apresentada no diagrama de Moran seja positiva, além de se detectar os valores discrepantes (outliers) e os pontos de alavancagem (leverage points).

Uma observação é considerada outlier, como já destacado anteriormente, quando não segue o mesmo processo de dependência espacial apresentado pelas demais, e é um ponto de alavancagem quando apresenta grande influência sobre a tendência central.

Para o estudo da função de produção da agropecuária do Rio Grande do Sul, foram analisadas as variáveis tradicionais: capital, trabalho e área; e para captar o grau de modernização da agropecuária inseriu-se a variável eletricidade. Foram também consideradas as diferenças edafoclimáticas dos municípios, de modo que se acrescentou a densidade pluviométrica (chuva) e a temperatura média anual. Para todas as análises utilizou-se a matriz de peso torre, uma vez que essa apresentou o maior coeficiente de I de Moran para o valor bruto da produção agropecuária.

O I de Moran bivariado, ${ }^{5}$ ou seja, o índice comparado entre duas variáveis do modelo é apresentado na Tabela 3. A variável endógena (valor bruto da produção agropecuária) é comparada com cada variável exógena do modelo. Como se observa, há evidência de autocorrelação positiva entre o valor bruto da produção e o capital, a área colhida, a energia elétrica e a chuva. Isso significa que municípios com altos (baixos) valores brutos da produção estão associados a municípios com altos (baixos) índices das variáveis consideradas (capital, área colhida, energia elétrica e chuva). Porém, deve-se destacar que a pseudo-significância da variável

$5 \quad$ O estudo do I de Moran bivariado, segundo Almeida (2004), tem como objetivo central descobrir se os valores de uma variável observada em uma região apresentam uma relação com os valores de uma outra variável observada nas regiões vizinhas. 
energia elétrica leva à interpretação de ausência de autocorrelação, uma vez que essa variável apresenta probabilidade não significativa. De modo antagônico, há evidência de autocorrelação negativa entre o valor bruto da produção e o trabalho e também entre o valor da produção e a temperatura, no entanto, a pseudo-significância dessa última associação é de $87 \%$, o que demonstra a ausência de autocorrelação entre as variáveis. A explicação para esses resultados está no fato de que os municípios com elevados (baixos) valores brutos da produção estão circundados por municípios com pequena (grande) área colhida, como se observou através do Mapa 1. O mesmo se aplica à análise da chuva, visto que os municípios com elevado (baixo) valor bruto da produção agropecuária estão associados a municípios com elevado (baixo) volume anual de chuvas, como se verifica nas regiões Norte e Nordeste do Estado, em municípios como Frederico Westphalen, Palmitinho, Entre Rios do Sul e Bento Gonçalves.

Tabela 3 - Coeficiente de I de Moran Bivariado do valor bruto da produção agropecuária do Rio Grande do Sul e demais variáveis

\begin{tabular}{c|c|c|c}
\hline Variáveis & E (I) & I & Probabilidade \\
\hline Capital & $-0,002$ & 0,0633 & 0,001 \\
\hline Trabalho & $-0,002$ & $-0,1106$ & 0,001 \\
\hline Área Colhida & $-0,002$ & 0,3569 & 0,001 \\
\hline Energia Elétrica & $-0,002$ & 0,0202 & 0,441 \\
\hline Chuva & $-0,002$ & 0,1213 & 0,001 \\
\hline Temperatura & $-0,002$ & $-0,0059$ & 0,874 \\
\hline
\end{tabular}

* pseudossignificância empírica baseada em 999 permutações aleatórias. Fonte: Elaboração dos autores.

A Figura 3 apresenta os diagramas de dispersão de Moran Bivariado para o valor bruto da produção agropecuária do Rio Grande do Sul e as demais variáveis explicativas. Através apenas da visualização dos diagramas não é possível se estabelecer correlação entre as variáveis em análise, uma vez que os pontos apresentados nos mesmos não seguem uma distribuição nítida entre os quadrantes.

Deve-se destacar que, embora os padrões globais de associação espacial possam estar também em consonância com os padrões locais, isso não ocorre necessariamente. De acordo com Almeida (2004), podem ocorrer dois casos distintos. O primeiro caso ocorre quando uma indicação de ausência de autocorrelação global oculta padrões de associação local. O caso oposto ocorre quando uma forte indicação de autocorrelação global pode ocultar padrões locais de associação. Por conseguinte, as estatísticas de autocorrelação global não têm capacidade de identificar a ocorrência 
de autocorrelação local estatisticamente significante. Logo, verifica-se a importância de se utilizar uma estatística que capte esse efeito.

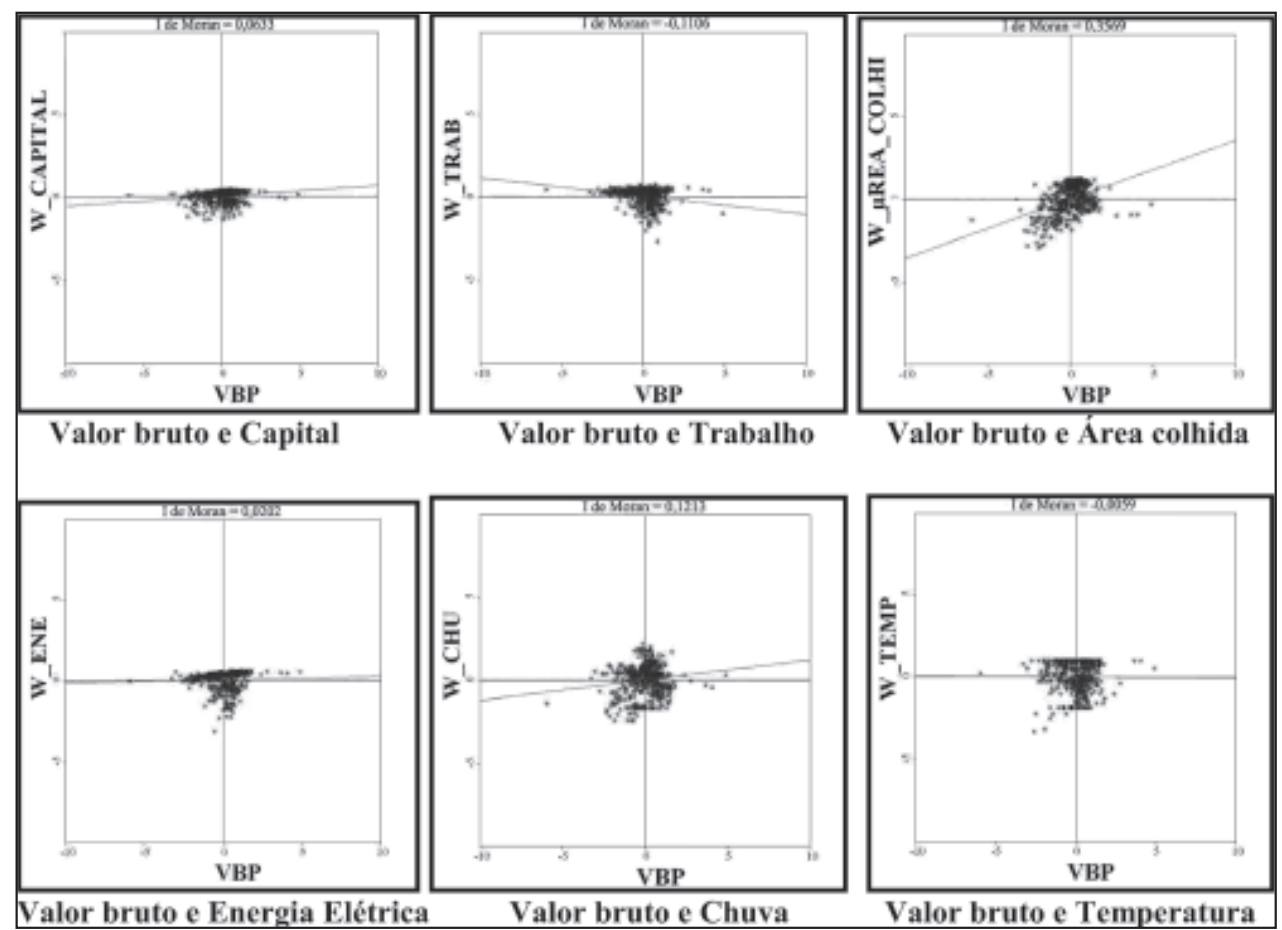

Figura 3 - Diagramas de Dispersão de Moran Bivariado para o valor bruto da produção agropecuária do Rio Grande do Sul e as demais variáveis explicativas Fonte: Elaboração dos autores.

\subsubsection{I de Moran Local}

Com capacidade de capturar padrões locais de associação linear, a estatística I de Moran local, segundo Almeida (2004), faz uma decomposição do indicador global de autocorrelação na contribuição local de cada observação em quatro categorias, cada qual correspondendo individualmente a um quadrante no diagrama de dispersão de Moran. A maneira mais evidente de especificação da estatística se dá por meio de mapas. O Mapa 3 apresenta a significância dos municípios considerando o valor bruto da produção agropecuária do Rio Grande do Sul.

Apresentando a formação de clusters, o Mapa 4 faz uma combinação entre as informações do diagrama de dispersão de Moran (Figura 2) e as informações do mapa de significância (Mapa 3), que considera as medidas de associação local do I de Moran e se dividem em quatro categorias de associação espacial estatisticamente significantes. São demonstrados os clusters que possuem significância estatística para o I de Moran local considerando a variável endógena do modelo. 


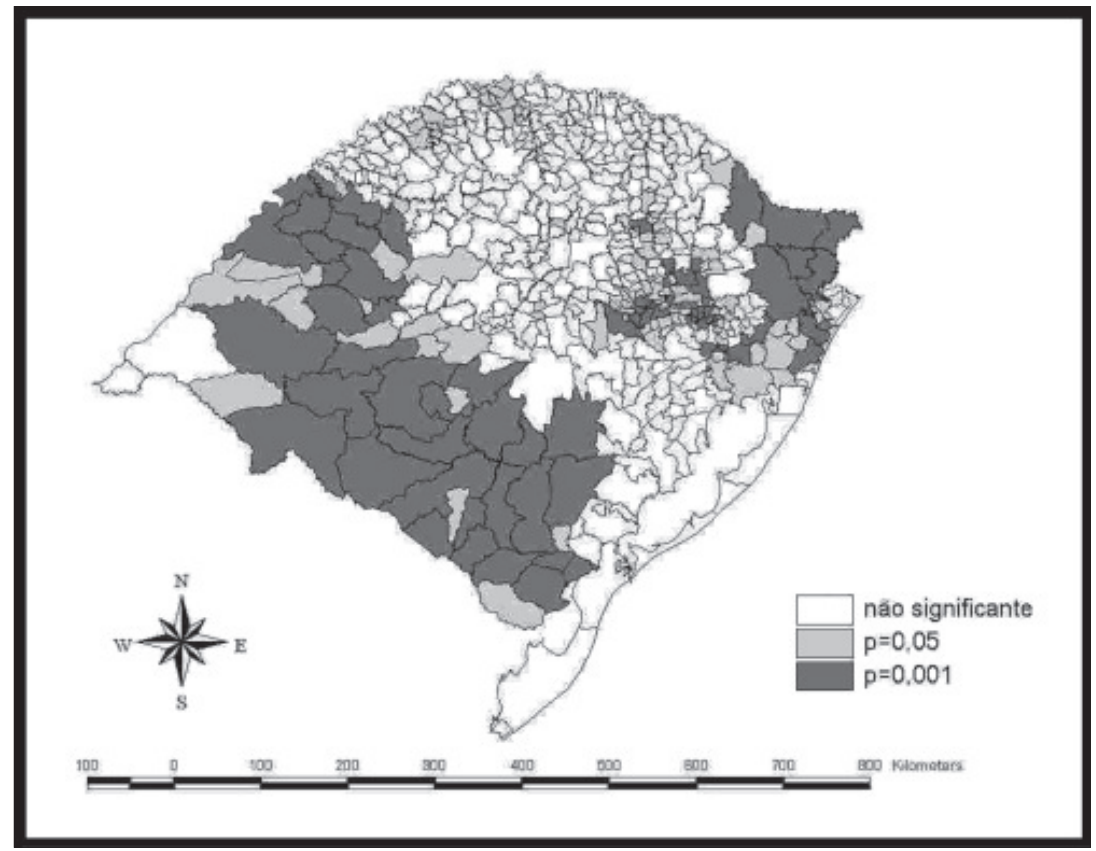

Mapa 3 - Significância do valor bruto da produção agropecuária do Rio Grande do Sul

Fonte: Elaboração dos autores.

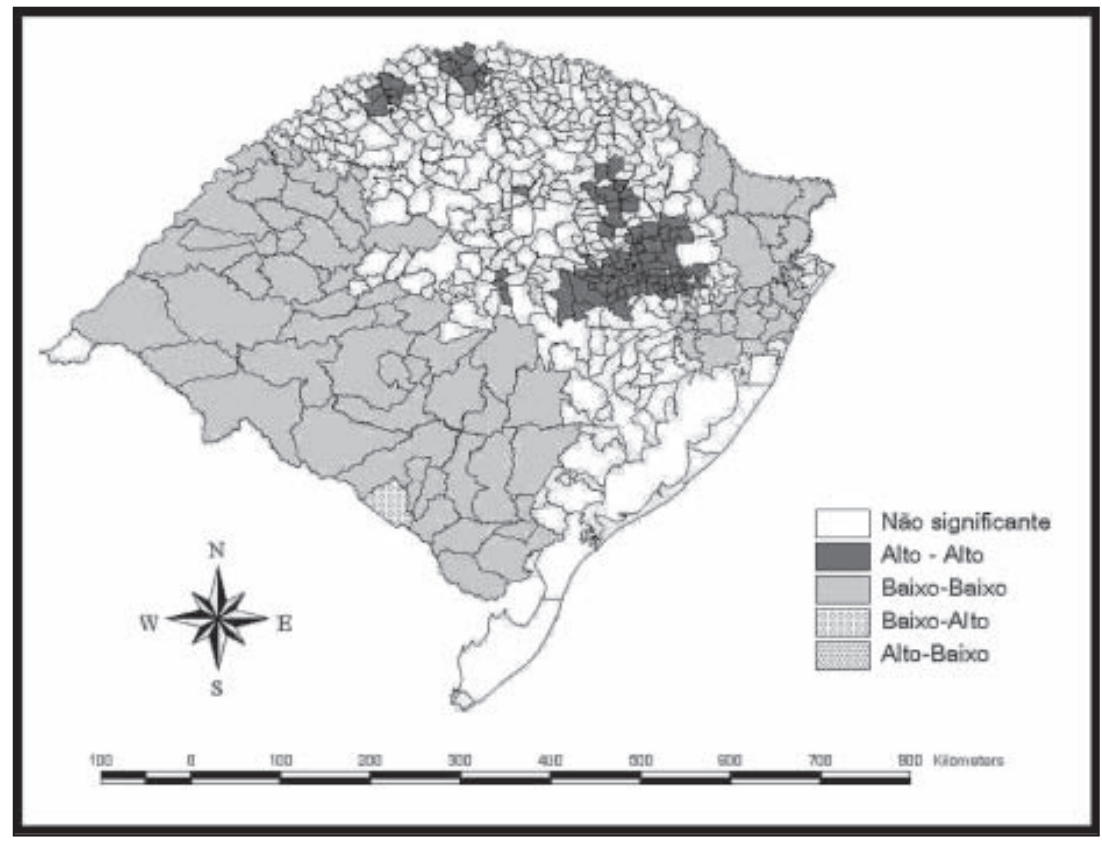

Mapa 4 - Clusters para valor bruto da produção agropecuária (Mil reais/ $\mathrm{Km}^{2}$ ) no Estado do Rio Grande do Sul, para o ano de 2004

Fonte: Elaboração dos autores. 
Através da figura é possível verificar a presença de dois clusters do tipo baixo-baixo, ${ }^{6}$ um com número elevado de municípios, situado na Região Sul do Estado, e outro localizado na Região Nordeste. O primeiro se caracteriza, de acordo com Alonso et al. (1994), pela predominância de grandes propriedades, da pecuária com criação de animais de grande porte, e também pela lavoura de arroz. Segundo os autores, o que se verifica na região é o desenvolvimento predominante da pecuária extensiva e da agricultura, porém destacando-se que em várias localidades as condições do solo não permitem uma maior diversificação das atividades. Isso ocorre devido à propensão à erosão que algumas áreas apresentam, enquanto outras não são ideais para uso de maquinários e implantação de lavouras temporárias.

O segundo cluster do tipo baixo-baixo, que abrange a região de Porto Alegre até municípios como São Francisco de Paula, Esmeralda, Muitos Capões, Vacaria e Bom Jesus, é caracterizado pela presença de vários setores industriais, além de grandes concentrações urbanas, segundo Alonso et al. (1994). Excetuando a região de Porto Alegre, tal cluster é formado por municípios com elevada extensão territorial, porém pobres, de acordo com Jansen e Waquil (2002). Tais características auxiliam na explicação do resultado obtido, ou seja, municípios que possuem baixo valor bruto da produção agropecuária e que são circundados por municípios com a mesma característica.

Há ainda três clusters do tipo alto-alto, ${ }^{7}$ sendo o maior localizado na Região Nordeste (Serra Gaúcha), e os demais na Região Norte. Com significativa importância para a economia do Estado, o cluster situado na Região Nordeste é formado por municípios como: Cotiporã, Veranópolis, Bento Gonçalves, Farroupilha, Caros Barbosa, dentre outros. Deve-se ressaltar que esses municípios são classificados como pequenos, desenvolvidos e relativamente rurais, conforme estudo realizado por Jansen e Waquil (2002). São caracterizados também pela fruticultura, em especial pelo cultivo da uva, assim como pela criação de animais de pequeno porte. $\mathrm{O}$ município de Bento Gonçalves, conhecido como a Capital Brasileira da Uva e do Vinho, é o maior produtor de uvas do Estado - 15,4\% da produção estadual (FEE, 2008) - e tem o setor como terceiro economicamente mais representativo no município. Destaca-se também o município de Farroupilha, responsável por aproximadamente 10\% de toda produção de uva do Estado.

$6 \quad$ Clusters do tipo baixo-baixo significam que os municípios que pertencem ao agrupamento possuem baixo valor bruto da produção agropecuária e são circundados por municípios que também possuem valores baixos para a mesma variável.

7 Clusters do tipo alto-alto significam que os municípios pertencentes a esse agrupamento exibem valores altos para a variável de interesse (valor bruto da produção agropecuária) e são rodeados por municípios que também apresentam valores altos. 
Através do mapa de clusters é possível medir a associação local entre as variáveis do modelo estudado. Segundo Almeida (2004), da mesma maneira que é possível obter um coeficiente de autocorrelação espacial global bivariado, pode-se alcançar uma medida de autocorrelação espacial local. Essa estatística apresenta o grau de associação linear (positiva ou negativa) entre o valor de uma variável em uma determinada região e a média de uma outra variável nas localizações vizinhas.

Os clusters formados pela associação entre o valor bruto da produção agropecuária (variável endógena da função de produção) e as variáveis exógenas do modelo (capital, trabalho, área colhida, energia elétrica, temperatura e chuva) são apresentados na Figura 4.

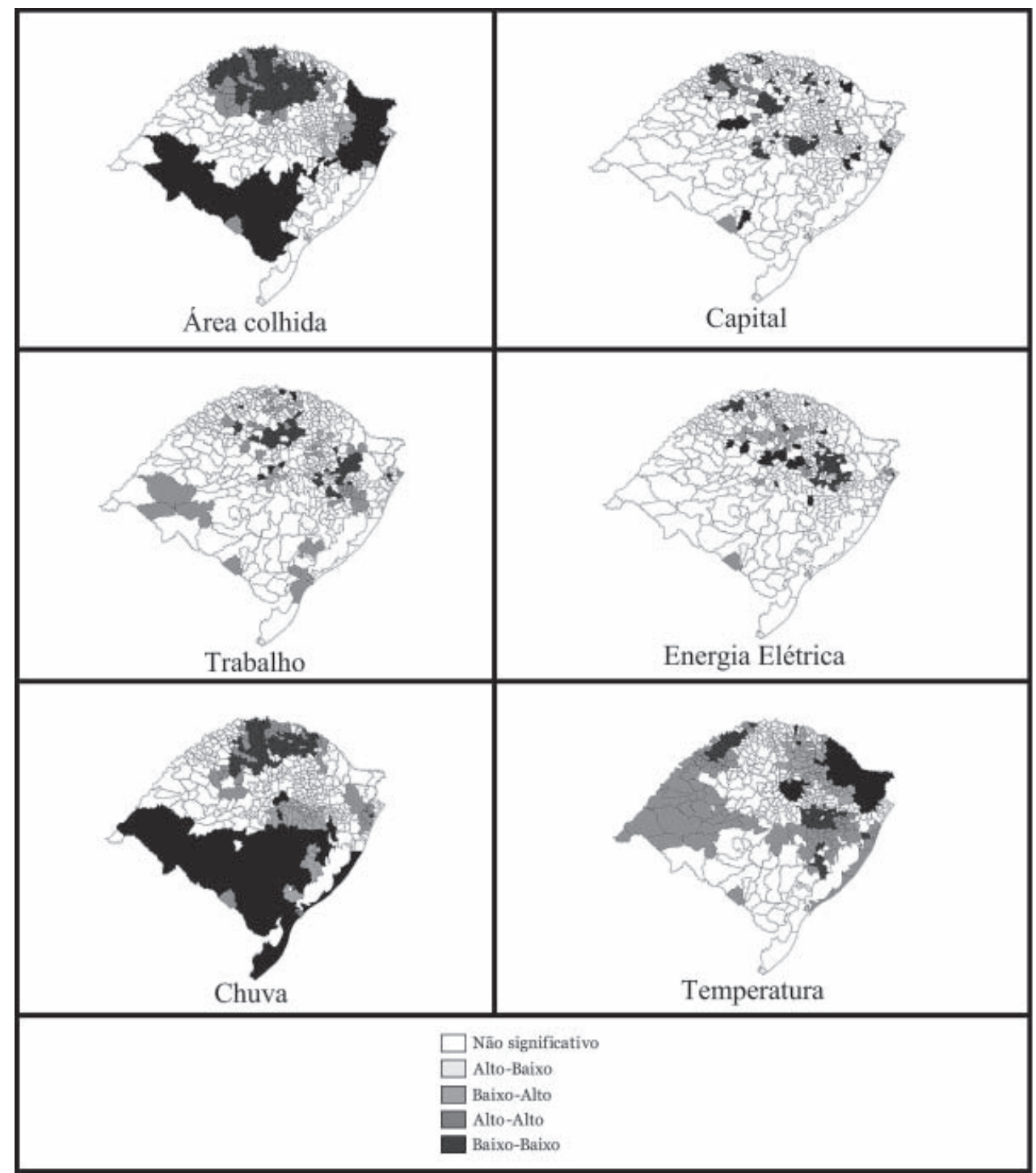

Figura 4 - Clusters Bivariados entre o valor bruto da produção agropecuária e as demais variáveis explicativas

Fonte: Elaboração dos autores. 
Ao analisar a associação entre o valor bruto da produção agropecuária e a área colhida, observa-se a presença de dois grandes clusters do tipo baixo-baixo, localizados nas Regiões Sul e Nordeste do Estado. Esse resultado pode ser interpretado pela presença de municípios com baixo valor da produção circundados por municípios com pequena área colhida. A existência de um cluster do tipo baixo-baixo na Região Sul se justifica pelo predomínio da criação de animais de grande porte, ou seja, da pecuária, de tal modo que a área colhida não é significativa. Verifica-se também a presença de um cluster do tipo alto-alto, localizado na Região Norte, o que evidencia a presença de municípios com elevado valor da produção associados a municípios que possuem elevada área colhida. Tais municípios se caracterizam pela produção de grãos, em especial soja, milho e trigo (JANSEN; WAQUIL, 2002).

Quando analisada a associação entre valor bruto da produção e capital, uma outra forma de distribuição pode ser observada. Verifica-se a presença de três pequenos clusters do tipo alto-alto, situados na região central e Norte do Estado. Isso ocorre devido à presença de municípios com elevado valor da produção que têm como vizinhos municípios com elevado capital. Tais municípios são predominantemente agrícolas e de pequena extensão territorial, de acordo com Alonso et al. (1994).

A associação entre o valor da produção e a variável trabalho apresenta-se bastante próxima à associação entre o valor da produção e a variável energia elétrica. Em ambas nota-se a presença de um cluster do tipo alto-alto na Região Nordeste, em municípios localizados na serra e que têm o cultivo da uva como uma das principais atividades econômicas. Tais municípios possuem elevado valor da produção agropecuária e são circundados por municípios com elevado consumo de energia elétrica e elevada utilização de mão-de-obra.

Os clusters obtidos pela associação entre o valor da produção e a variável chuva se localizam em regiões distintas do Estado. Cabe salientar que o Rio Grande do Sul é marcado por baixa incidência de chuvas ao Sul e o inverso ao Norte, o que explica a formação de um cluster do tipo baixo-baixo na Região Sul e outro do tipo alto-alto na Região Norte.

Por fim, observa-se na associação entre o valor bruto da produção e a variável temperatura a presença de sete diferentes clusters. Esse resultado se explica pelas diferentes temperaturas observadas no Estado, sendo a Região Nordeste a mais fria, com temperatura média anual entre $12^{\circ} \mathrm{C}$ e $16^{\circ} \mathrm{C}$, e a Região Oeste a mais quente, com temperatura média anual variando entre $18^{\circ} \mathrm{C}$ e $22^{\circ} \mathrm{C}$ (SEMC, 2008). 


\section{Considerações Finais}

Considerando os objetivos propostos pelo trabalho de analisar a distribuição espacial da agropecuária no Estado do Rio Grande do Sul no ano de 2004, verificando a existência de clusters, e examinar o nível de correlação entre as variáveis contidas na função de produção, pôde-se constatar, mediante a Análise Exploratória de Dados Espaciais, uma maior concentração de municípios com elevado valor bruto da produção agropecuária nas regiões Norte e Nordeste do Estado. Esses municípios se caracterizam pela pequena dimensão territorial e pela diversidade na produção agrícola.

Ao analisar a distribuição do valor bruto da produção no Estado, levando-se em consideração a presença de municípios tidos como outliers, ou seja, municípios com valores discrepantes do restante do Estado, verificou-se a presença de 11 municípios que não seguem o mesmo processo de dependência espacial dos demais, 5 considerados outliers altos e 6 outliers baixos. Os municípios considerados outliers altos situam-se nas regiões Norte e Nordeste do Estado e são caracterizados pela pequena dimensão territorial e pelo elevado valor da produção agropecuária.

As estimativas do I de Moran global evidenciaram haver autocorrelação espacial positiva entre os municípios gaúchos para o valor bruto da produção agropecuária. Isto significa que os municípios com elevado (baixo) valor bruto da produção são circundados por municípios com elevado (baixo) valor da mesma variável.

Ao inserir as variáveis exógenas do modelo (capital, trabalho, área, energia, chuva e temperatura) na estimativa do I de Moran global verificouse que as variáveis capital, área colhida e chuva, apresentaram autocorrelação espacial positiva com a variável valor da produção, e a variável trabalho apresenta autocorrelação espacial negativa. As demais variáveis, energia elétrica e temperatura, não apresentaram um padrão significativo de autocorrelação espacial com o valor bruto da produção. Esses resultados se devem principalmente às características peculiares do Estado, onde os municípios com elevado valor da produção possuem pequena extensão territorial, baixas temperaturas, nível elevado de precipitação e pequeno número de trabalhadores.

Para verificar os padrões locais de associação, estimou-se o I de Moran local e constatou-se a presença de dois clusters do tipo baixo-baixo, localizados nas Regiões Sul e Nordeste do Estado, e três clusters do tipo alto-alto, localizados na Região Nordeste (Serra Gaúcha), onde há o predomínio do cultivo da uva, e dois na Região Norte.

Analisando os clusters formados pela associação entre o valor bruto da produção e as demais variáveis exógenas, pôde-se observar diferentes 
níveis de associação entre os municípios, destacando-se a presença de um cluster do tipo alto-alto para a associação com a área colhida, localizado na Região Norte, que se caracteriza pela presença de municípios com elevado valor bruto da produção circundados por municípios com grandes áreas colhidas, apesar da pequena extensão territorial de tais municípios. Essa associação demonstra a existência de elevada produtividade no cultivo agrícola de grãos, principal atividade dos municípios e cujo valor de comercialização é bastante significativo.

A inclusão da atividade pecuária no estudo da função de produção mostrou-se importante, uma vez que os resultados da pesquisa melhoraram significativamente. Conforme assinalam Rocha e Parré (2008), a pecuária apresenta-se como um importante setor na geração de valor adicionado para a economia do Estado do Rio Grande do Sul e sua inserção no estudo espacial da agricultura pode propiciar uma análise mais eficiente.

Considerando os resultados obtidos, que demonstram a diversidade pela qual o Estado é marcado, torna-se importante a execução de políticas que busquem atender as necessidades específicas dos municípios e das regiões as quais pertencem. Deve-se ressaltar que a realização dessas políticas também proporcionará efeitos positivos às localidades que circundam tais municípios devido às características associativas que apresentam entre si.

\section{Referências}

ALONSO, J. A. F.; BENETTI, M. D.; BANDEIRA, P. S. Crescimento Econômico da Região Sul do Rio Grande do Sul: Causas e Perspectivas. Porto Alegre: FEE, 1994.

ALMEIDA, E. S. Curso de Econometria Espacial Aplicada. Piracicaba, 2004.

. Função de Produção Agropecuária Espacial. In: Congresso Brasileiro de Economia e Sociologia Rural, 41, Ribeirão Preto. Anais... Ribeirão Preto: Sociedade Brasileira de Economia e Sociologia Rural (SOBER), CD-ROM, 2005.

BATISTA, I. M.; SILVEIRA, V. C. P.; ALVES, F. D. As Desigualdades Econômicas Regionais e o Setor Agropecuário do Rio Grande do Sul. In: XLIV Congresso da Sociedade Brasileira de Economia e Sociologia Rural, Fortaleza. XLIV Congresso da Sociedade Brasileira de Economia e Sociologia Rural, 2006.

CASTRO, N. Custos de Transporte e Produção Agropecuária no Brasil, 1970-1996. Agricultura em São Paulo, v. 49, n. 2, São Paulo, p. 87-109, 2002.

FEE - FUNDAÇÃO DE ECONOMIA E ESTATÍSTICA DO RIO GRANDE DO SUL. Disponível em < http://www.fee.rs.gov.br > . Acesso em: jan. 2008.

GOMES, A. P.; BAPTISTA, A. J. M. S. Função de Produção de Fronteira e Tomada de Decisão na Agropecuária. In: Congresso Brasileiro de Economia e Sociologia Rural, 42, Cuiabá. Anais... Cuiabá: Sociedade Brasileira de Economia e Sociologia Rural (SOBER), CD-ROM, 2004.

IBGE - INSTITUTO BRASILEIRO DE GEOGRAFIA E ESTATÍSTICA - PAM - Pesquisa Agrícola Municipal. Disponível em: <http://www.ibge.gov.br>. Acesso em: dez. 2007. 
.IBGE-Cidades@. Disponível em: <http://www.ibge.gov.br/cidadesat/default.php>. Acesso em: jan. 2008.

IPEA - INSTITUTO DE PESQUISA ECONÔMICA APLICADA. Boletim de Conjuntura no 78. Disponível em: <http://www.ipea.gov.br>. Acesso em: set. 2007.

IPEA - IPEADATA - Moeda e Crédito. Disponível em: <http://www.ipeadata.gov.br>. Acesso em: mar. 2008.

JANSEN, S. L.; WAQUIL, P. D. Identificação e Caracterização das Atividades Agropecuárias nos Municípios Gaúchos: Uma comparação com indicadores sócio-econômicos. In: 40 Congresso Brasileiro de Economia e Sociologia Rural, Passo Fundo. Anais XL Congresso Brasileiro de Economia e Sociologia Rural, 2002.

PEROBELLI, F. S.; ALMEIDA, E. S.; ALVIM, M. I. S.; FERREIRA, P. G. C. A. Análise Espacial da Produtividade do Setor Agrícola Brasileiro: 1991-2003. In: Congresso Brasileiro de Economia e Sociologia Rural, 43, Ribeirão Preto. Anais... Ribeirão Preto: Sociedade Brasileira de Economia e Sociologia Rural (SOBER), CD-ROM, 2005.

PIMENTEL, E. A.; ALMEIDA, L. O.; SABBADINI, R. Comportamento Recente das Exportações Agrícolas no Brasil: Uma Análise Espacial no Âmbito dos Estados. In: Congresso da Sociedade Brasileira de Economia e Sociologia Rural, 43, Ribeirão Preto. Anais... Ribeirão Preto: Sociedade Brasileira de Economia e Sociologia Rural (SOBER), CD-ROM, 2005.

RAIS - RELAÇÃO ANUAL DE INFORMAÇÕES SOCIAIS. Disponível em: < http://www. mte.gov.br >. Acesso em: mar. 2008.

ROCHA, C. B.; PARRE, J. L. Setor Agrícola Gaúcho: Um Estudo de Sua Distribuição Espacial. In: XXIII Semana do Economista (UEM), Maringá. Anais... XXIII Semana do Economista Alimentos e Energia: Crise ou Oportunidade?, CD-ROM, 2008.

SEMC - SECRETARIA DE INFRA-ESTRUTURA E LOGÍSTICA DO RIO GRANDE DO SUL. Disponível em: <http://www.semc.rs.gov.br/> . Acesso em: mai. 2008.

SEPLAG - SECRETARIA DO PLANEJAMENTO E GESTÃO DO RIO GRANDE DO SUL. Atlas Socioeconômico do Rio Grande do Sul. Disponível em: < http://www.scp.rs.gov.br/ ATLAS >. Acesso em: mai. 2008.

SIMM, E. B.; ALVES, A. F. Concentração Espacial da Agroindústria Paranaense Durante a Década de 1990. In: Congresso Brasileiro de Economia e Sociologia Rural, 43, Ribeirão Preto. Anais... Ribeirão Preto: Sociedade Brasileira de Economia e Sociologia Rural (SOBER), CD-ROM, 2005.

SOUZA E SILVA, S. S. Logística Aplicada à Colheita Mecanizada de Cereais. São Paulo, maio, 2004. Disponível em: <http://www.teses.usp.br/teses/disponiveis/11/11148/tde08102004-153330/publico/saulo.pdf>. Acesso em: mar. 2008.

Recebido em: 23/07/2008.

Aceito em: 05/09/2008. 